\title{
Informational Lobbying and Political Contributions
}

\author{
Bennedsen, Morten; Feldmann, Sven E.
}

Document Version

Final published version

Publication date:

2000

License

CC BY-NC-ND

Citation for published version (APA):

Bennedsen, M., \& Feldmann, S. E. (2000). Informational Lobbying and Political Contributions.

Link to publication in CBS Research Portal

\section{General rights}

Copyright and moral rights for the publications made accessible in the public portal are retained by the authors and/or other copyright owners and it is a condition of accessing publications that users recognise and abide by the legal requirements associated with these rights.

\section{Take down policy}

If you believe that this document breaches copyright please contact us (research.lib@cbs.dk) providing details, and we will remove access to the work immediately and investigate your claim. 


\title{
Institut for Nationaløkonomi
}

Handelshøjskolen i København

Working paper 8-2000

\section{INFORMATIONAL LOBBYING AND POLITICAL CONTRIBUTIONS}

\author{
Morten Bennedsen Sven E. Feldmann
}

Department of Economics - Copenhagen Business School

Solbjerg Plads 3, DK-2000 Frederiksberg 


\title{
Informational Lobbying and Political Contributions
}

\author{
Morten Bennedsen* and Sven E. Feldmann**
}

June 2000

\begin{abstract}
Interest groups can influence political decisions in two distinct ways: by offering contributions to political actors and by providing them with relevant information that is favorable for the group. We analyze the conditions under which interest groups are more inclined to use one or the other channel of influence. First, we identify an indirect cost of searching for information in the form of an information externality that increases the cost of offering contributions. We then show that an extreme interest group might find it beneficial to abandon information provision altogether and instead seek influence solely via contributions. Finally, we apply our model to cast doubt on the "conventional wisdom" that competition among information providers increases the amount of information provided: when the identified information externality is taken into account, we show that competition decreases information search. Thus, our analysis lends support to a rather cynical view of lobbying wherein lobby groups provide little or no useful information to the political process.
\end{abstract}

\footnotetext{
*Department of Economics, Copenhagen Business School and CIE. Email: MB.ECO@CBS.DK

**University of Chicago, Email: SvEN@UCHICAGO.EDU

We would like to thank Eric Maskin, Torsten Persson, and Jim Snyder for valuable comments. The first author gratefully acknowledges financial support from the Danish Social Science Research Council and Industriens Realkreditfond.
} 


\section{Introduction}

Interest groups participate in the political decision making process in two distinct ways: they provide substantive information to the policy maker, and they offer financial incentives by contributing to politicians' campaigns. ${ }^{1}$ Before any particular policy decision an interest group thus faces a choice between providing the decision maker with information or lobbying via contributions, or doing both, in order to achieve a favorable outcome. This paper analyzes how the choices of acquiring information and providing it to the decision maker and supporting her campaign interact, and under what circumstances an interest group uses one or both of these two different strategies.

In the framework of this paper both the interest group and the decision maker are uncertain about some aspect of a policy decision. Depending on the true nature of the uncertain aspect, the decision maker prefers either an outcome that favors the interest group or one that harms it. The interest group has the ability to gather information that may reduce the uncertainty, and it may therefore be in the position to provide the decision maker with useful information. Obviously, the interest group will only gather and transmit the information if it is in its interest to do so. Alternatively, the interest group may take advantage of the decision maker's ignorance and induce her to choose the favorable outcome by offering (contingent) campaign contributions.

Collecting information and deciding not to provide it to the decision maker is information in and of itself, and a rational decision maker will make use of it. Independently of whether the interest group's search for information is observed by the decision maker, the collection of information can create an informational externality, particularly when it leads the decision maker to infer that the group is knowledgeable and is withholding its information. This information externality increases the cost of bribing the decision maker.

\footnotetext{
${ }^{1}$ In addition to influencing politicians directly, interest groups may try to influence voters by providing information or cues to them via advertisement or endorsements. This aspect of interest group behavior does not concern us in this paper.
} 
We prove that if the lobby group has intense preferences for a particular outcome (as most interest groups indeed have), then the information externality significantly decreases the lobby group's incentive to search for information.

It is often argued that competition among information providers increases the incentive to provide (truthful) information (see e.g. Austen-Smith and Wright 1992 and Dewatripont and Tirole 1999). This "conventional wisdom" no longer holds without qualification when the information externality we identified is taken into account. We show that as long as interest groups do not have the ability to offer campaign contributions, competition indeed increases information search. Allowing for contributions, however, reverses the result: competition among information providers decreases an extreme interest group's incentive to engage in information provision.

An important feature of our analysis is that the decision maker cannot enter a binding contract in which she commits ex ante to a policy choice conditional on the lobby group's search activity or the search outcome. In other words, the decision maker always retains the right to choose the ex post optimal outcome. This incomplete contracting framework implies that the outcome that maximizes joint surplus may not be achievable, in particular if this outcome requires that the interest group reveal information that is detrimental to its own goals. In our view, the absence of contracting distinguishes interest groups from (government) agencies or contractors, where delegation of decision authority is conceivable. ${ }^{2}$

Campaign contributions as well as the strategic use of information to influence policy choice have been the subject of tremendous scholarly interest and research (see surveys by Schlozman and Tierney 1987 and by Persson 1998). A growing number of formal models assess the role of campaign contributions and party or candidate competition for interest group influence on policy outcomes (Austen-Smith 1987; Baron 1989, 1994; Snyder 1990, 1991; Grossman and Helpman 1994, 1996). In these models, contributions

\footnotetext{
${ }^{2}$ Let us emphasize that it is only the decision maker who lacks this commitment ability. In the vein of most lobbying literature we assume that interest groups can commit to nonnegative contributions or bribes conditioned on policy outcomes.
} 
serve as an incentive device with which interest groups induce policy choices. Ball (1995), on the other hand, considers a model in which contributions are welfare enhancing and serve as a signalling device. Extreme interest groups are willing to pay higher contributions so that a decision maker who cares about the welfare of the interest group can in a separating equilibrium be informed about the interest group's type.

A second strand of literature addresses the role of strategic information provision. Information originating from interested parties is biased; yet by using costly signals or sporadic verification of the information by the decision maker, the interest group can inform the decision maker to some extent (Calvert 1985, Gilligan and Krehbiel 1987). The influence it has on the decision maker provides the interest group with an incentive to acquire costly information.

Few papers have combined the motivations to provide campaign contributions and information. Austen-Smith (1995) considers a two-stage model in which in the first stage contributions serve to gain access and partially signal the group's preference, followed by a second stage game of costless information provision. Lohmann (1995) analyzes the situation where several heterogeneous interest groups seek access and influence. Interest groups with preferences similar to the decision maker's obtain access at zero cost and can transmit private information credibly, while extreme lobby groups must pay positive contributions. In both models contributions serve to gain access and to enhance the credibility of the sender.

The present paper takes a different approach. It contrasts the cynical view of interest groups as influence seekers by means of contributions with the more optimistic view of interest groups as providers of useful information. By allowing interest groups to choose between the two means of influence (and any combination of the two) the paper demonstrates the interaction between the incentives to offer contributions and to supply information.

The next section lays out a simple generic decision model used in the subsequent sections. In Section 3, which serves as a brief warm-up and provides the basic intuition for the following sections, we make the simplifying 
but unrealistic assumption that the decision maker observes the lobby group's search activity.

Sections 4 and 5 present the two main contributions of our analysis in the more realistic setting where the decision maker does not monitor the interest group's search activity. Section 4 shows that allowing an interest group to use financial incentives decreases its search activity for a large range of the parameters. In Section 5 we introduce a competing information provider and ask if competition among information providers changes the lobby group's incentive to gather information. We show that in our model this competition increases the lobby's search activity when contributions are not permitted, but decreases the lobby's search activity when contributions are allowed.

Section 6 discusses the findings and concludes.

\section{The Model}

A decision maker needs to choose a project $d \in\left\{d_{\ell}, d_{\circ}, d_{r}\right\}$. Whether $d_{\ell}$ or $d_{r}$ is the best choice depends on the state of the world, $\theta \in\left\{\theta_{\ell}, \theta_{r}\right\}$, while $d_{\circ}$ is a "neutral" choice. The state of the world is unknown to the decision maker, and each state has an equal probability. ${ }^{3}$

The decision maker holds preferences over the projects and, in addition, derives utility from potential contributions from an interest group.

$$
V_{d m}(d, c ; \theta)=u_{d m}(d ; \theta)+g c
$$

We assume that the utility is risk averse over the projects, ${ }^{4}$ and additively separable and linear in the contributions $c$, with $g$ as parameter measuring the (marginal) effectiveness of campaign contributions in utility terms. ${ }^{5}$ For

\footnotetext{
${ }^{3}$ The symmetry of the probability between the states is not important for what follows, but it simplifies the notation and analysis.

${ }^{4}$ Risk averse over the projects in the sense that the decision maker strictly prefers $d_{\circ}$ to an even gamble $\left(\frac{1}{2}, \frac{1}{2}\right)$ between $d_{\ell}$ and $d_{r}$.

${ }^{5}$ This means that the decision maker is risk neutral with respect to contributions. If the decision maker were risk averse, contingent contributions could influence the decision maker additionally by providing an insurance.
} 
simplicity of exposition we choose an explicit functional form for $u$ as follows:

$$
u_{d m}(d ; \theta)=\left\{\begin{array}{rll}
0 & \text { if } & (d, \theta) \in\left\{\left(d_{\ell}, \theta_{\ell}\right),\left(d_{r}, \theta_{r}\right)\right\} \\
-1 & \text { if } & d=d_{\circ} \\
-4 & \text { if } & (d, \theta) \in\left\{\left(d_{\ell}, \theta_{r}\right),\left(d_{r}, \theta_{\ell}\right)\right\}
\end{array}\right.
$$

There is one interest group in the basic model, and it prefers the $d_{\ell}$ project independently of the state of the world. We thus use $\ell$ as subscript for the for the group's utilities. The lobby group's preference is additively separable in the utility from the project, the cost of gathering private information, and the contributions it pays to the decision maker:

$$
\begin{aligned}
V_{\ell}(d, s, c) & =u_{\ell}(d)-s k-c \\
\text { where } u_{\ell}(d) & =\left\{\begin{array}{rll}
2 \beta & \text { if } & d=d_{\ell} \\
1 & \text { if } & d=d_{\circ} \\
0 & \text { if } & d=d_{r} .
\end{array}\right.
\end{aligned}
$$

$k \geq 0$ is the cost incurred by the group if it decides to search for information, $s \in\{0,1\} . \beta \geq \frac{1}{2}$ parameterizes the lobby group's intensity of preference between the projects. Notice that the group is risk averse (risk loving) if $\beta<(>) 1$. We believe that $\beta>1$ is the leading case, reflecting the fact that interest groups often have a lot to gain from the choice of their most preferred outcome. We will refer to such interest groups as having intense preferences.

The specific functional forms for the decision maker's and the group's preferences are chosen for their simplicity and the flexibility they afford. The decision maker essentially has a quadratic loss function over evenly spaced alternatives, while the interest group's preference intensity is expressed by the parameter $\beta$.

The contractual framework between interest group and decision maker is incomplete. We assume that the decision maker and the lobby group are unable to write binding ex ante contracts that tie the decision maker's choice to the lobby's search activity or to the information transmitted by the group, or that require payments by the decision maker to the lobby group contingent on the chosen policy. We believe this to be a defining aspect of 
lobby groups. It would be politically infeasible and practically problematic to bind the decision maker to the lobby group's recommendation. If the decision maker were to make such a commitment, the information provider becomes a (government) agency or contractor. ${ }^{6}$

The sequence of moves in the game applies both to lobbying in a democratic society through legal campaign contributions to political parties and to corruption through bribes in a bureaucratic regime. In the first stage of the game the lobby group decides whether it wishes to search for information about the state of the world. If the group searches for information, it receives a signal $\sigma$, which with probability $q$ contains hard information about the state of the world, $\sigma=\theta$, and with probability $1-q$ is uninformative, $\sigma=\emptyset$. Next, the interest group sends message $m$ to the decision maker, where $m \in\{\sigma, \emptyset\}$. Notice that the group can withhold knowledge about the state of the world, but it cannot "lie", i.e. it cannot forge the information. If $m=\emptyset$ it may either be that the group is uninformed or that it is not willing to provide the information it has.

In addition to sending a message, the interest group can also lobby by offering contributions, understood as non-negative payments to the decision maker contingent on the chosen project (similar to Bernheim and Whinston 1986). Finally, given messages and contingent contributions, the decision maker chooses the project.

The time line is given in Figure 1.

When the interest group searches it receives a favorable signal $\sigma=\theta_{\ell}$ with probability $\frac{1}{2} q$, and will reveal this to the decision maker. If the lobby group receives bad information $\left(\sigma=\theta_{r}\right)$, it will hide this from the decision maker and will send the same message as if its search was without any success $(\sigma=\emptyset)$. The decision maker's posterior belief following the message $m=\emptyset$ is: $p\left(\theta_{r} \mid m=\emptyset\right)=\frac{1}{2-q}$, and $p\left(\theta_{\ell} \mid m=\emptyset\right)=\frac{1-q}{2-q}$.

\footnotetext{
${ }^{6}$ In addition, we also assume that commitment to a third party, e.g. the public, is not enforceable. The consequences of contracting with third parties are analyzed in Maskin and Tirole 1999.
} 


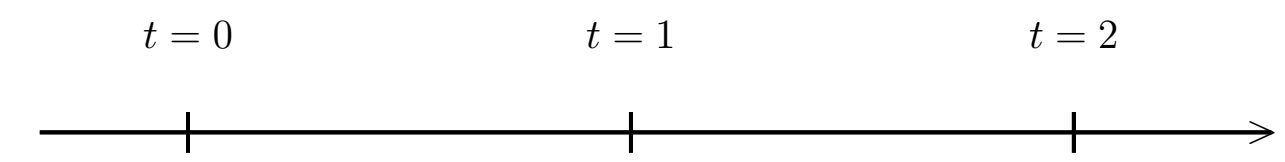

Lobby group chooses Lobby group sends whether to search message and offers or not contributions

Decision maker chooses project

Figure 1: Time Line

Before we begin the formal analysis, it is useful to establish under what condition a lobby induces $d_{\ell}$ via contributions even after it has searched (or the decision maker believes it has searched) and failed to provide hard evidence in favor of $\theta_{\ell}$. We generally refer to such groups as extreme lobby groups. Define

$$
\underline{\beta}(q) \equiv \begin{cases}\frac{1}{2}+\frac{1+\frac{1}{2} q}{(2-q) g} & \text { if } q<\frac{2}{3} \\ \max \left\{\frac{2 q}{(2-q) g} ; \frac{1}{2}+\frac{1+\frac{1}{2} q}{(2-q) g}\right\} & \text { otherwise. }\end{cases}
$$

$\underline{\beta}(q)$ as lower bound for the intensity of an extreme lobby group's preferences, as stated formally in Lemma 1.

Lemma 1. Suppose the decision maker knows (or believes) that the lobby has searched for information, and the group fails to provide any hard evidence. Then a lobby group induces its preferred outcome through contribution if and only if $\beta \geq \underline{\beta}(q)$.

The proof is in the appendix. The Lemma characterizes when a lobby group who cares sufficiently for its preferred outcome would use contributions in a second attempt to sway a decision maker after the information search failed. 


\section{Observable Information Gathering}

This section identifies the Bayesian information externality associated with the lobby groups search. For simplicity and to provide a clear intuition we assume that the decision maker observes the lobby's search activity. In the subsequent sections we analyze the more realistic situation where the decision maker cannot monitor the interest group.

For now assume contributions are not feasible (e.g. they are illegal or non-enforceable). When the decision maker receives $m=\theta_{j}$ she chooses $d=d_{j}(j=\ell$ or $r)$, as the message is necessarily true. If the message is uninformative $(m=\emptyset)$, the decision maker updates her beliefs and chooses the project that maximizes expected utility, which is $d_{\circ}$ if $q<\frac{2}{3}$, and $d_{r}$ if $q>\frac{2}{3}$, given the updated beliefs. With this optimal strategy for the decision maker, will the group search for information?

First, consider the case of $q<\frac{2}{3}$. The lobby group searches if the expected gain from searching exceeds the (certain) payoff from not searching, or $\frac{q}{2} 2 \beta+$ $\left(1-\frac{q}{2}\right) \cdot 1-k>1$, which simplifies to

$$
\frac{q}{2}(2 \beta-1)>k
$$

Notice that if search is costless $(k=0)$ the group will search in this case.

Next, consider $q>\frac{2}{3}$, i.e. the search is likely to be informative for the group, and the decision maker switches to $d_{r}$ if $m=\emptyset$. The group now searched if $\frac{q}{2} 2 \beta-k>1$, or

$$
\frac{q}{2}(2 \beta-1)>k+\left(1-\frac{q}{2}\right)(1-0)
$$

The new term on the right hand side is the expected indirect search cost, or Bayesian cost, and arises since the rational decision maker updates her beliefs whenever no information is provided by a searching lobby.

If search is costless $(k=0)$, the condition simplifies to $\beta>\frac{1}{q}$ and only a group with sufficiently intense preference for its preferred outcome finds it optimal to search. The condition is decreasing in $q$ and reaches a lower 
bound of $\beta=1$, where search is perfectly informative $(q=1)$. Thus, the bound is strictly higher than when $q<\frac{2}{3}$. We get the following result:

Result 1 (Indirect search cost). When search is observable and is sufficiently informative $\left(q \geq \frac{2}{3}\right)$, a lobby group only searches for information if its preference for the preferred outcome is sufficiently intense. Specifically, the group searches iff $\beta>\frac{1+k}{q}$.

Let us now introduce campaign contributions as monetary incentives. The lobby group has the dual choice of searching for information and how to influence the decision maker's choice via contributions. In contrast to the previous case without contributions, even for $q<\frac{2}{3}$ and costless search a lobby with intense preferences does not always want to search when the opportunity for contributions is available. The result is rather strong and applies for all extreme lobby groups. The proof is in the appendix.

Result 2 (No search I). Assume that search is observable, that contributions are allowed, and that the lobby group's preferences are sufficiently intense $(\beta>\underline{\beta}(q))$. Then in equilibrium the lobby never searches for information.

The result states that if the group knows ex ante which decision it wants to induce, then it will never search for information, even if searching is costless. While the group could save contributions if the search reveals the favorable state of the world, this is dominated by the fact that the information externality created by its search makes contributions more costly if the search is not successful.

Notice that when contributions are feasible, the indirect cost of information collection takes on a different form than when contributions are not feasible. In the previous section, the indirect cost was an increase in the likelihood of state $\theta_{r}$ that leads the decision maker to choose $d_{r}$, the worst outcome for the group. When contributions are feasible, however, the indirect cost of searching is the increase in expected contributions that are necessary to induce the lobby groups's favored outcome. Result 2 states that the benefit of searching never outweighs the (indirect) cost. 
Following the logic of Result 2, interest groups with intense preferences, e.g. the tobacco industry or the beneficiary from protective tariffs, will not seriously try to find evidence that supports their claims, but rather simply lobby for the outcome they prefer. Only groups with less intense preferences, who choose not to induce their most preferred decision in the absence of hard information to support their view, will gather relevant information.

The result does not depend on the functional forms chosen, but is a general feature of this information game. ${ }^{7}$ The result is a consequence of the information externality created by the group's search: the group's searchwhether successful or not - increases the information available to the decision maker at a later stage of the game and thereby increases her expected utility. As the final decision by assumption does not depend on the outcome of the group's search, the total surplus created by the decision is fixed, which implies that the lobby group must be worse off.

\section{Unobservable Information Gathering}

Due to transaction costs and possible lack of incentives it seems realistic that the decision maker does not observe the interest group's search activity. When search is unobservable, we must solve for a rational expectation equilibrium (REE), in which the decision maker correctly infers from the lobby group's equilibrium behavior its decision to search, and both players' strategies maximize their expected utilities given their beliefs.

We begin the analysis by assuming contributions are not feasible. We first show the existence of rational expectation equilibria in which the lobby group does not search.

Suppose the decision maker expects the lobby group not to search. If the lobby group provides no information $(m=\emptyset)$, the decision maker does

\footnotetext{
${ }^{7}$ Result 2 is robust to assuming for example that the two states of the world $\theta_{\ell}$ and $\theta_{r}$ have different probabilities as long as the decision maker chooses the neutral outcome when no search is performed. It is also robust to assuming that the decision maker is risk neutral or risk loving since in this case the decision maker is willing to choose the lobby's preferred outcome at no cost.
} 
not update her beliefs and chooses the neutral outcome. The lobby group's net benefit from searching given that the decision maker expects no search ${ }^{8}$ is $\frac{q}{2} 2 \beta+\left(1-\frac{q}{2}\right) \cdot 1-k-1$. The equilibrium condition is that, given the beliefs, the lobby group prefers not to search, i.e. that the expression above is negative, which can be written as

$$
\begin{aligned}
\frac{q}{2}(2 \beta-1) & <k \\
\text { or } \quad \beta & <\frac{1}{2}+\frac{k}{q} .
\end{aligned}
$$

Thus, if the the lobby group's preference is not too intense there exists a rational expectation equilibrium in which the group does not search whenever search cost is positive. The higher the search cost and the lower the informational value of the search $(q)$, the easier it is to sustain a no-search equilibrium. However, if search is costless $(k=0)$, then the lobby group will always search and no REE without search can be supported.

The condition for searching is the same as in the observable case with $q<\frac{2}{3}$; neither situation implies an indirect cost of search since the absence of information does not affect the decision maker's policy choice. Thus, the condition just states that for the group to search, the benefit of searching must exceed the effective direct cost of searching.

Next, we solve for equilibria in which the group searches. Thus, suppose the decision maker expects the lobby group to search. We need to consider two cases, depending on the size of $q$.

If $q<\frac{2}{3}$ the decision maker chooses the neutral outcome in the absence of any information. The lobby group's incentive to search is therefore precisely as above, and there exists a rational expectation equilibrium with search if and only if $\beta>\frac{1}{2}+\frac{k}{q}$.

If $q>\frac{2}{3}$ the decision maker chooses $d_{r}$ unless the lobby group offers hard information for $\theta_{\ell}$. The condition for searching now becomes,

$$
\frac{q}{2} 2 \beta+\left(1-\frac{q}{2}\right) \cdot 0>k,
$$

\footnotetext{
${ }^{8}$ Since a successful search reveals hard information about $\theta$ the question of the decision maker's out-of-equilibrium belief does not arise.
} 
Observable search:

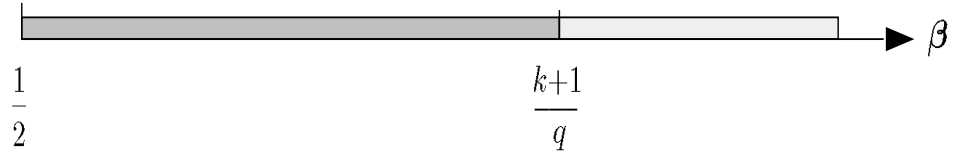

Unobservable search:

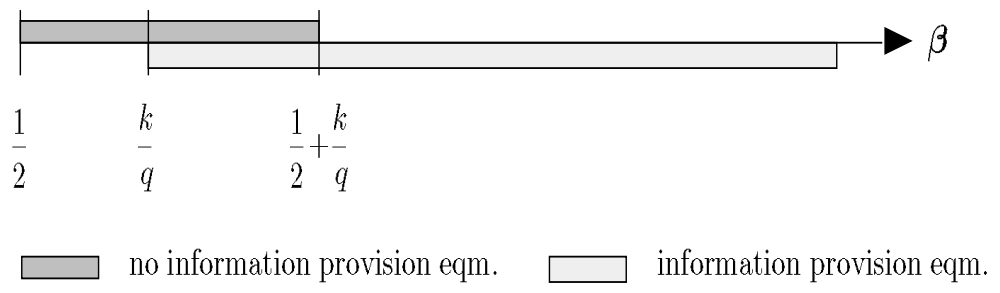

Figure 2: Incentives to search when no contributions are permitted and $q>$ $2 / 3$.

$$
\text { or } \quad \beta>\frac{k}{q} \text {. }
$$

By comparing equations (1) and (2) we notice several things: first, if the lobby group's preference is extreme or if the direct cost of searching is small enough, there is a unique REE in which the group searches for information; second, if the preference is not extreme or the direct search cost is very high, there is a unique REE without search; and finally, in between these there exists a range of preferences and search costs where we have multiple rational expectation equilibria. The condition for multiple equilibria is

$$
\beta \in\left[\frac{k}{q}, \frac{1}{2}+\frac{k}{q}\right] .
$$

Figure 2 illustrates the result and compares the incentives to provide information when search is observable versus non-observable and contributions are not feasible for $q>\frac{2}{3}$. Comparing the two cases we see that in the range $\left[\frac{1}{2}+\frac{k}{q}, \frac{k+1}{q}\right]$ the group does not gather any information when search is observable, while it must do so in a rational expectations equilibrium when search 
is non-observable. Furthermore, when $\beta$ is in the range $\left[\frac{k}{q}, \frac{1}{2}+\frac{k}{q}\right]$ there is a REE where the decision maker expects the lobby group to search and the lobby group has an incentive to do so, and one where the decision maker expects no search and the lobby group has no incentive to deviate. Which equilibrium is played depends only on the expectation of the decision maker. Notice that the group prefers not to search, as becomes clear by looking at the case where the group can commit not to search, i.e. when search is observable.

We can make some further observations: if $k<\frac{1}{3}$ searching is always a possible REE, and as the cost for searching vanishes $(k=0)$, searching becomes the unique REE in the non-observable search case. The latter is not true when search is observable.

To summarize: there is clearly more search and information provision in equilibrium when the search activity is not observable, as the group has an incentive to search if the decision maker cannot directly observe it, and a group with intense preferences is more likely to search.

Let us now introduce contributions into the analysis. Again, assume that the lobby group is extreme so that it always wishes to induce its preferred policy through either information provision or contributions, i.e. that $\beta>$ $\underline{\beta}(q)$. Which means will such an extreme lobby group employ in order to achieve its favored outcome?

Result 3. Assume that search is unobservable, that contributions are allowed, and that the lobby group is extreme $(\beta>\underline{\beta}(q))$. Let

$$
\bar{k}=\frac{q}{2 g} \quad \text { and } \quad \overline{\bar{k}}= \begin{cases}\frac{q}{2 g}\left(\frac{2+q}{2-q}\right) & \text { if } q<\frac{2}{3} \\ \frac{q}{2 g}\left(\frac{4 q}{2-q}\right) & \text { otherwise. }\end{cases}
$$

i) If the direct search cost is lower than $\bar{k}$, then there is a unique rational expectation equilibrium (REE) in which the lobby group searches for information and provides this information if it is in its favor, otherwise offers contribution as given in equation (6). 
ii) If the direct search cost is higher than $\overline{\bar{k}}$, the unique REE implies that there is no search, and the group offers contributions $c=\frac{1}{g}$ ex ante.

iii) If the direct search cost is higher than $\bar{k}$ but lower than $\overline{\bar{k}}$, then there is a search and a no-search REE, together with contribution offers as in (i) and (ii), respectively. There also exists a mixed strategy equilibrium, characterized in the appendix.

The proof of Result 3 is given in the appendix. The result describes in what situation the lobby group chooses to provide information and when it uses contributions to induce its preferred outcome, assuming that the group's search is a private activity. $\bar{k}$ is the lowest direct search cost consistent with the existence of an equilibrium in which the lobby group does not search for information. $\overline{\bar{k}}$ is the highest direct search cost consistent with the existence of an equilibrium in which the lobby group searches for information.

Comparing Result 3 with the analysis in Section 3, more information is gathered and provided when search is not observable than when it is publicly observable. The group has a greater incentive to search when it cannot be monitored, and the decision maker takes this into account. She expects the group to search as long as the search cost is not too high $(k<\bar{k})$, and multiple equilibria can be supported for an intermediate range of search costs $(\bar{k}<k<\overline{\bar{k}})$ depending on the decision maker's belief about the group's behavior.

How does the use of contributions affect the lobby group's incentives to collect information when the decision maker cannot observe the groups' search activity? Figure 3 compares the sets of equilibria for the two cases.

The figure assumes an extreme lobby group $(\beta>\underline{\beta}(q))$. Panel A depicts the case where $q<\frac{2}{3}$ and Panel B the case of $q>\frac{2}{3}$. The difference is that in the absence of hard evidence for $\theta_{\ell}$ the decision maker chooses $d_{\circ}$ in Panel A while she chooses $d_{r}$ in Panel B.

Suppose first that contributions are not feasible. Above we proved that when $q<\frac{2}{3}$ then the lobby searches if and only if $\beta>\frac{1}{2}+\frac{k}{q}$, which is the area above the upward sloping line in in Panel A. In Panel B both search and non- 


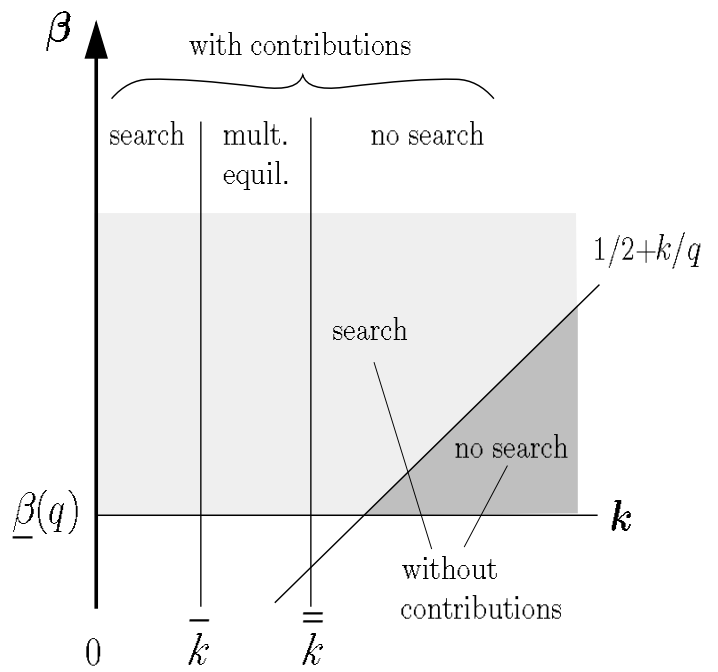

Panel A: $q<2 / 3$

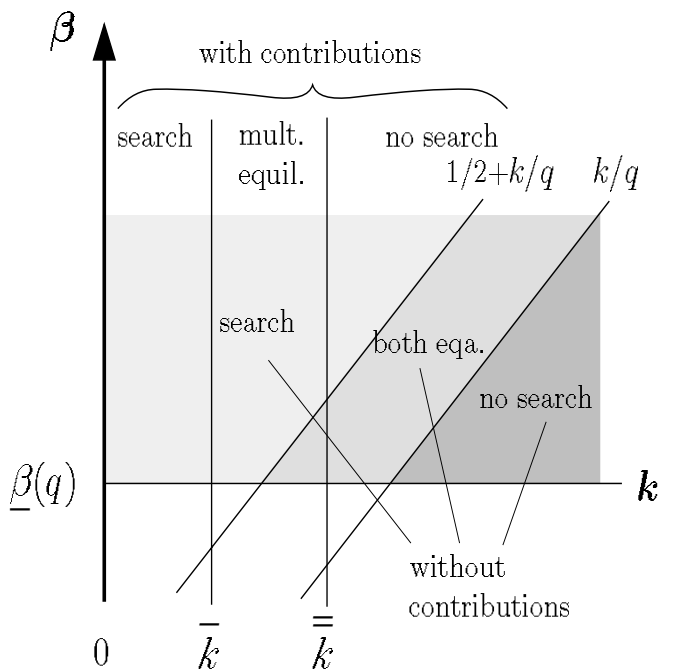

Panel B: $q>2 / 3$

Figure 3: Information Provision and Contributions

search can be sustained in a rational expectation equilibrium if $\beta \in\left[\frac{k}{q}, \frac{1}{2}+\frac{k}{q}\right]$. Notice that when the lobby cannot make contributions, it searches whenever the preferences are intense enough relative to the effective search cost. Since information provision is the only way to induce the decision maker to choose the lobby's preferred outcome, it is natural that an intense lobby group is willing to pay high search costs, whereas a lobby with less intense preferences is not.

Next, suppose the lobby group can make contributions. As shown in Result 3, if the direct search cost is lower than $\bar{k}$ (higher than $\overline{\bar{k}}$ ), there is a unique searching (no-searching) equilibrium and in between these bounds there are multiple equilibria, where both search and no-search can be sustained in equilibrium. These areas are drawn in Panel A and B. Notice that when the lobby has both instruments to induce its preferred outcome, it engages in information provision whenever the direct search cost is relatively 
small and abstains from it whenever this cost is large.

In the area above the $\beta>\frac{1}{2}+\frac{k}{q}$ line and to the right of $\overline{\bar{k}}$, allowing campaign contributions strictly decreases the lobby group's provision of information. In this area the lobby has intense preferences and is willing to incur high search cost in order to affect the decision maker's choice if this is the only means of influence. However, when the lobby group can choose between contribution and information provision, it prefers to use contributions only. This is credible even though search is unobservable because the search cost is sufficiently high. Notice that for a given search cost $k$ above $\overline{\bar{k}}$, if preferences are sufficiently extreme then the introduction of the contribution instrument always decreases the amount information provision. Hence, Result 2 essentially holds in the case of unobservable search when search is sufficiently costly:

Result 4 (No Search II). Assume that search is unobservable, that the lobby group's preferences are sufficiently intense $\left(\beta>\max \left\{\underline{\beta}(q), \frac{1}{2}+\frac{k}{q}\right\}\right)$, and that search is sufficiently costly $(k>\overline{\bar{k}})$. Then the possibility of offering contributions strictly reduces the interest group's search activity in a rational expectation equilibrium.

The result supports the main insight of this paper: extreme lobby groups may lack incentives to provide information if they have alternative ways to induce the decision maker to chose the lobby's favored outcome. When contributions are not allowed, an intense lobby group is willing to incur higher direct search cost because they gain more from successful information provision. However, this argument hinges crucially on the absence of the possibility of making contributions and, therefore, on the absence of any indirect cost of collecting information on the required contributions. Once contributions are allowed, this indirect cost, which are represented in the decision maker's updating of her beliefs when the information collection is unsuccessful, dominates the gain from inducing the lobby's favored outcome through successful information collection.

To establish this result we need Lemma 2, which shows that Figure 3 is 
indeed correct. Notice in Panel A that the intersection between $\beta=\underline{\beta}(q)$ and the $\beta=\frac{1}{2}+\frac{k}{q}$ line is to the right of $\overline{\bar{k}}$. This implies that allowing contributions cannot increase search when $q<\frac{2}{3}$. Similarly in Panel B, the intersection between $\beta=\underline{\beta}(q)$ and the $\beta=\frac{1}{2}+\frac{k}{q}$ line is to the right of $\bar{k}$ and the intersection between $\beta=\underline{\beta}(q)$ and $\beta=\frac{k}{q}$ is to the right of $\overline{\bar{k}}$. This implies that whenever interest groups want to induce their preferred outcome $(\beta>\underline{\beta}(q))$, if no-search is the unique equilibrium when contributions are not permitted, then search cannot be an equilibrium when contributions are allowed. Similarly, if no-search is an equilibrium without contributions, then it is also an equilibrium when contributions are feasible. Lemma 2 proves that this is always the case.

\section{Lemma 2.}

a) Let $q<\frac{2}{3}$. Then $\beta>\underline{\beta}(q)$ and $k<\overline{\bar{k}}$ implies that $\beta>\frac{1}{2}+\frac{k}{q}$.

b) Let $q>\frac{2}{3}$. (i) $\beta>\underline{\beta}(q)$ and $k<\overline{\bar{k}}$ implies that $\beta>\frac{k}{q}$. (ii) $\beta>\underline{\beta}(q)$ and $k<\bar{k}$ implies that $\beta>\frac{1}{2}+\frac{k}{q}$.

Proof. a) $\frac{1}{2}+\frac{k}{q}<\frac{1}{2}+\frac{1}{2 g}\left(\frac{2+q}{2-q}\right)<\beta$ where the first inequality uses the definition of $\overline{\bar{k}}$ and the second uses the definition of $\underline{\beta}(q)$. b) (i) $\frac{k}{q}<\frac{\overline{\bar{k}}}{q}=\frac{2 q}{(2-q) g}<\beta$. (ii) $\beta>\frac{1}{2}+\frac{1+\frac{1}{2} q}{(2-q) g}>\frac{1}{2}+\frac{1}{g}>\frac{1}{2}+\frac{\bar{k}}{q}>\frac{1}{2}+\frac{k}{q}$.

\section{Competition Among Information Providers}

A standard result in the information based lobbying literature holds that competition among information providers increases the incentive for each lobby to provide information. We apply our model to multiple information providers to study how robust this result is in the presence of monetary contributions.

We assume that the decision maker contacts a third agent, whom we will call a researcher. As part of his daily work, this researcher has a positive private benefit of being involved in information collection. We assume that the researcher has biased preferences in the sense that he strictly prefers one of the outcomes independently of the state of the world. Hence, even 
though the researcher always collects information, he may - similar to the interest group — withhold this information from the decision maker if it does not support his preferred choice. Furthermore, we assume that the researcher is wealth constrained and cannot make contributions to the decision maker or the lobby group. ${ }^{9}$

The timing is as follows: At date 0 the researcher searches for information. At the same time the lobby group also has the opportunity to search for information. At date 1 the two agents send their messages to the decision maker. After observing the researcher's message the lobby group can submit a contribution offer to the decision maker conditional on the decision maker's choice. ${ }^{10}$ At date 2 the decision maker chooses the outcome given the messages and taking into account the contributions from the lobby group.

We study the case where the decision maker, who cannot monitor the interest group's search activity, approaches a researcher who, independently of the state of the world, prefers the outcome $d_{r}$, that is, the researcher and the lobby group have conflicting interests. In the following we use subscript $\ell$ and $r$ to refer to the lobby group and the researcher respectively.

\section{The decision maker's beliefs}

The researcher searches for information and receives a signal $\sigma_{r}$. With probability $q_{r}$ the researcher's signal is informative and reveals the true state of the world $\theta$. The researcher reveals this information to the decision maker whenever it is favorable for him, i.e. if $\sigma_{r}=\theta_{r}$. He sends the message $m=\emptyset$ whenever he is uninformed or knows that $\theta=\theta_{\ell}$.

If the decision maker does not expect the lobby group to search, her

\footnotetext{
${ }^{9}$ Our discrete policy model is too simple to analyze the case of two identical lobbies, since it would reduce to a matter of which group was most extreme and most wealthy. Notice that this simplified model still gives the "standard" result, that competition increases information provision, when contributions are not allowed. We, therefore, conjecture that generalizing to two symmetric interest groups will not change the result.

${ }^{10}$ Information collection may be investing in research projects and therefore take a long time. We believe it is reasonable to assume that the lobby group can make the contribution offer in the last moment before the decision is taken and after the observable messages are sent.
} 
posterior belief after receiving $m_{r}=\emptyset$ is

$$
\begin{aligned}
p\left(\theta_{\ell} \mid m_{r}=\emptyset\right) & =\frac{1}{2-q_{r}} \\
\text { and } \quad p\left(\theta_{r} \mid m_{r}=\emptyset\right) & =\frac{1-q_{r}}{2-q_{r}} .
\end{aligned}
$$

If $q_{r}>\frac{2}{3}$, the lobby group has no incentive to search, since the decision maker chooses the lobby's preferred outcome whenever the researcher does not provide any information. Hence, to make this application interesting we assume in the following that $q_{r} \leq \frac{2}{3}$.

Table 1: Types and likelihoods of messages sent by the lobby group and the researcher in each true state of the world and depending on the outcome of the agents' information collection.

True state:

\begin{tabular}{l|c|c|c|}
\cline { 2 - 4 } & & $\theta_{\ell}$ & $\theta_{r}$ \\
\cline { 2 - 4 } & $\sigma_{\ell}=\sigma_{r}=\emptyset$ & $m_{\ell}=m_{r}=\emptyset$ & $m_{\ell}=m_{r}=\emptyset$ \\
& $\frac{1}{2}\left(1-q_{\ell}\right)\left(1-q_{r}\right)$ & $\frac{1}{2}\left(1-q_{\ell}\right)\left(1-q_{r}\right)$ \\
\cline { 2 - 4 } $\begin{array}{l}\text { Result of } \\
\text { information } \\
\text { collection: }\end{array}$ & $\sigma_{\ell}=\theta, \sigma_{r}=\emptyset$ & $m_{\ell}=\theta, m_{r}=\emptyset$ & $m_{\ell}=m_{r}=\emptyset$ \\
\cline { 2 - 4 } & $\sigma_{\ell}=\emptyset, \sigma_{r}=\theta$ & $\frac{1}{2} q_{\ell}\left(1-q_{r}\right)$ & $\frac{1}{2} q_{\ell}\left(1-q_{r}\right)$ \\
\cline { 2 - 4 } & & $\frac{1}{2}\left(1-m_{\ell}=\emptyset\right.$ & $m_{\ell}=\emptyset, m_{r}=\theta$ \\
& & $\frac{1}{2}\left(1-q_{\ell}\right) q_{r}$ \\
\hline \multirow{2}{*}{$\sigma_{\ell}=\sigma_{r}=\theta$} & $m_{\ell}=\theta, m_{r}=\emptyset$ & $m_{\ell}=\emptyset, m_{r}=\theta$ \\
& & $\frac{1}{2} q_{\ell} q_{r}$ & $\frac{1}{2} q_{\ell} q_{r}$ \\
\hline
\end{tabular}

Next, assume that the lobby searches and that the decision maker believes that both the researcher and the lobby search for information. Table 1 summarizes the types and likelihoods of messages sent from the two agents to the decision maker in each true state of the world and depending on the outcome of the information collection. ¿From this table we calculate the decision maker's posterior beliefs when she does not receive any information:

$$
\begin{aligned}
p\left(\theta_{\ell} \mid m_{\ell}=m_{r}=\emptyset\right) & =\frac{1-q_{\ell}}{2-q_{\ell}-q_{r}} \\
\text { and } \quad p\left(\theta_{r} \mid m_{\ell}=m_{r}=\emptyset\right) & =\frac{1-q_{r}}{2-q_{\ell}-q_{r}} .
\end{aligned}
$$


When $q_{r} \leq \frac{2}{3}$ and in the absence of any contributions from the lobby group, the cutoff level for $q_{\ell}, \bar{q}_{\ell}\left(q_{r}\right)$, at which the decision maker switches between $d_{\circ}$ and $d_{r}$ if she receives no hard information from either agent, is

$$
\begin{array}{rlrl}
u_{d m}\left(d_{\circ}\right)-u_{d m}\left(d_{r}\right) & =0 \\
\Leftrightarrow & -1-(-4) \frac{1-q_{\ell}}{2-q_{\ell}-q_{r}} & =0 \\
\Leftrightarrow & q_{\ell}=\frac{2+q_{r}}{3} & \equiv \bar{q}_{\ell}\left(q_{r}\right) .
\end{array}
$$

The decision maker therefore chooses $d_{\circ}$ if $q_{\ell}<\bar{q}_{\ell}\left(q_{r}\right)$ and $d_{r}$ if $q_{\ell}>\bar{q}_{\ell}\left(q_{r}\right)$. Notice that $\bar{q}_{\ell}\left(q_{r}\right)>\frac{2}{3}$, which is the point at which the decision maker switches in the absence of an additional information source.

\section{No contributions}

To replicate the "conventional wisdom," which holds that competition among information providers increases information provision, we begin by disallowing contributions.

If the decision maker expects that the lobby does not search, then the lobby has no incentive to search iff:

$$
\begin{aligned}
\frac{q_{\ell}}{2} 2 \beta+\left(1-\frac{q_{\ell}}{2}\right) 1-k-1 & <0 \\
\Leftrightarrow \quad \beta & <\frac{1}{2}+\frac{k}{q_{\ell}} .
\end{aligned}
$$

Next, assume that the decision maker expects the lobby to search. If $q_{\ell}<$ $\bar{q}_{\ell}\left(q_{r}\right)$, the decision maker chooses $d_{\circ}$ in the absence of any evidence and the lobby's search condition is again given by equation (4), that is the lobby group searches if and only if $\beta>\frac{1}{2}+\frac{k}{q_{\ell}}$. If $q_{\ell}>\bar{q}_{\ell}\left(q_{r}\right)$, the decision maker chooses $d_{r}$ in the absence of any evidence and the lobby's search condition becomes:

$$
\begin{array}{rlrl}
\frac{q_{\ell}}{2} 2 \beta+\left(1-\frac{q_{\ell}}{2}\right) 0-k-1 & >0 \\
\Leftrightarrow \quad & \beta & >\frac{k}{q_{\ell}} .
\end{array}
$$


Inspecting equations (4) and (5), we notice that for $q_{\ell}>\bar{q}_{\ell}\left(q_{r}\right)$ there are multiple equilibria whenever

$$
\beta \in\left[\frac{k}{q_{\ell}}, \frac{1}{2}+\frac{k}{q_{\ell}}\right] .
$$

Comparing the search conditions above with the ones found in Section 4, we observe that if $q_{\ell} \in\left[\frac{2}{3}, \bar{q}_{\ell}\left(q_{r}\right)\right]$ and $\beta \in\left[\frac{k}{q_{\ell}}, \frac{1}{2}+\frac{k}{q_{\ell}}\right]$ then the introduction of an alternative information provider may increase the lobby group's search activity when search is unobservable and contributions are not feasible.

This result indicates that competition between information providers increases the information given to the decision maker. Thus, we have replicated the standard result previous derived by e.g. Austen-Smith and Wright 1992 and Dewatripont and Tirole 1999 in somewhat different settings. The intuition is that the lobby's indirect cost of information collection decreases when an agent with conflicting preferences also searches. However, as we show in the following, this result hinges crucially on the assumption that monetary transfers are not feasible.

\section{Allowing for contributions}

We now allow the lobby group to make contributions to the decision maker. As usual we focus on an extreme lobby group with $\beta>\underline{\beta}(q)$.

When the decision maker receives no hard evidence and expects that the lobby group is not searching, the required contribution from the lobby group to induce its preferred outcome, $C_{\text {No Search }}$, is,

$$
C_{\text {No Search }}=\left(\frac{1-q_{r}}{2-q_{r}} 4-1\right) \frac{1}{g}=\frac{2-3 q_{r}}{\left(2-q_{r}\right) g} .
$$

We have not assumed that the lobby group will induce its preferred outcome through contributions when the researcher finds positive evidence against this outcome. For this reason, let $\delta$ be a dummy variable that takes the value 1 whenever the lobby group's preferences are so extreme that it induces its preferred outcome even if the decision maker knows that the true state of the world is $\theta_{r}$ (i.e. if $\beta>\frac{2}{g}$ ) and zero if not (i.e. $\beta<\frac{2}{g}$ ). Using this dummy, we 
can write the condition for the lobby group to abstain from searching given that the decision maker does not expect it to search:

$$
\begin{array}{rlrl}
\frac{q_{\ell}}{2} 2 \beta+\left(1-\frac{q_{\ell}}{2}-\frac{q_{r}}{2}\right) & \left(2 \beta-\frac{2-3 q_{r}}{\left(2-q_{r}\right) g}\right)+\delta\left(\frac{q_{r}}{2}\left(2 \beta-\frac{4}{g}\right)\right)-k \\
& <\left(1-\frac{q_{r}}{2}\right)\left(2 \beta-\frac{2-3 q_{r}}{\left(2-q_{r}\right) g}\right)+\delta\left(\frac{q_{r}}{2}\left(2 \beta-\frac{4}{g}\right)\right) \\
\Leftrightarrow & k & >\frac{q_{\ell}}{2 g}\left(\frac{2-3 q_{r}}{2-q_{r}}\right) \equiv \bar{k}_{r} .
\end{array}
$$

$\bar{k}_{r}$ is the lower bound on the cost of searching for supporting an equilibrium without search. Comparing this with the lower bound $\bar{k}$ in the model without the alternative information channel defined in Result 3, we observe that $q_{r}>0$ implies that $\bar{k}_{r}<\bar{k}$ for all $q_{\ell} \in[0,1]$.

Next, let the decision maker expect the lobby group to search. If the decision maker receives no hard evidence, the necessary contribution, $C_{\text {Search }}$, for the lobby group to induce its preferred outcome is,

$$
C_{\text {Search }}= \begin{cases}\frac{2+q_{\ell}-3 q_{r}}{\left(2-q_{\ell}-q_{r}\right) g} & \text { if } q_{\ell}<\bar{q}_{\ell}\left(q_{r}\right) \\ \frac{4\left(q_{\ell}-q_{r}\right)}{\left(2-q_{\ell}-q_{r}\right) g} & \text { otherwise. }\end{cases}
$$

The lobby group therefore searches if and only if

$$
\begin{aligned}
& \frac{q_{\ell}}{2} 2 \beta+\left(1-\frac{q_{\ell}}{2}-\frac{q_{r}}{2}\right)\left(2 \beta-C_{\text {Search }}\right)+\delta\left(\frac{q_{r}}{2}\left(2 \beta-\frac{4}{g}\right)\right)-k \\
& >\left(1-\frac{q_{r}}{2}\right)\left(2 \beta-C_{\text {Search }}\right)+\delta\left(\frac{q_{r}}{2}\left(2 \beta-\frac{4}{g}\right)\right) \\
& \Leftrightarrow \quad k<\overline{\bar{k}}_{r} \equiv \frac{q_{\ell}}{2} C_{\text {Search }}= \begin{cases}\frac{q_{\ell}}{2 g} \frac{2+q_{\ell}-3 q_{r}}{2-q_{\ell}-q_{r}} & \text { if } q_{\ell}<\bar{q}_{\ell}\left(q_{r}\right) \\
\frac{q_{\ell}}{2 g} \frac{4\left(q_{\ell}-q_{r}\right)}{2-q_{\ell}-q_{r}} & \text { otherwise. }\end{cases}
\end{aligned}
$$

$\overline{\bar{k}}_{r}$ is the upper bound on search cost for supporting an equilibrium with search. Comparing this to the similar cut point $\overline{\bar{k}}$ in the model without an alternative channel of information defined in Result 3, we observe that $\overline{\bar{k}}_{r}<\overline{\bar{k}}$ for all $q_{\ell} \in[0,1]$ and $q_{r} \in\left[0, \frac{2}{3}\right]$.

This yields the following result: 


\section{Result 5 (Less Search with Competing Information Providers).}

The introduction of a competing information provider increases the set of pure strategy equilibria in which the extreme lobby group does not search, and decreases the set of equilibria in which the extreme lobby group searches for information.

Above, it was shown that when the lobby groups are prohibited from making contributions, the presence of an extra agent increases the group's incentives to provide information. However, we have now shown that this "conventional wisdom" hinges crucially on the absence of contributions: when we allow the lobby group to make contributions, the introduction of an competing information provider decreases the lobby group's incentive to search for information.

The intuition is that the introduction of the competing information channel decreases both the cost and the benefit of engaging in information collection. The indirect search cost is lower because the decision maker's updating that occurs when the lobby group searches unsuccessfully is moderated when neither agent finds hard evidence in support of their respective favored outcome. However, the introduction of the researcher also reduces the lobby group's benefit from searching for information. This benefit is the amount of saved contribution when it finds supporting evidence for its preferred outcome. The reduction in this benefit arises because the lobby, if not searching, has to bribe less, since the decision maker updates her beliefs against the researcher. When the lobby is not allowed to use contributions, it only experiences the reduction in the indirect search cost and, therefore, searches more. When contributions are permitted, the lobby also faces the reduced benefit from searching and, as Result 5 shows, the reduction in the benefit of searching dominates the reduction in the indirect cost of searching, so that

the introduction of this competing information provider reduces the lobby's incentive to search. 


\section{Discussion}

Interest groups often have privileged access to information and are thus in the position to provide a political decision maker with information that is crucial for making the correct decision. At the same time, interest groups arguably have the ability to influence the decision maker's choice via financial incentives in form of campaign contributions. The models we presented in this paper show under what circumstances interest groups have the incentive to collect and provide such information, or to use contributions.

When equipped with the ability to make contributions, the group weights the direct and indirect costs of searching against the cost of inducing a favorable outcome via contributions to the decision maker. However, we point out that an unsuccessful search partially informs the decision maker and thereby raises the cost of inducing the group's preferred outcome ex post. The information externality, or indirect search cost, is thus reflected in higher contributions. A strong implication is formalized in Result 2 under the simplifying assumption, that the lobby's search activity is observable: for groups with a strong enough preference for their preferred outcome - groups we call extreme - the indirect search cost always dominates the benefit of getting the most preferred outcome 'for free' by providing information. Thus, extreme lobby groups never search and rather use contributions to induce their preferred decision.

The ability to offer contributions thus conflicts with the group's willingness to search and to provide useful information to the policy maker. The subsequent results show that the implications first derived in the simplified model continue to hold when search is unobservable. If search activity is unobservable, a rational decision maker will still have equilibrium knowledge of whether (or with what probability) the group searched by taking into account the lobby group's incentive to search. Interest groups search and provide information in equilibrium only if the cost of searching is lower than a threshold, independently of their preference intensity. Result 3, which characterizes the rational expectations equilibria of this game, shows that groups 
with intense preferences strictly prefer contributions as means to achieve their preferred outcome whenever a high enough search cost makes the abstention from search credible. Result 4 strengthens the implication and shows that allowing for contributions strictly reduces the set of equilibria in which the interest group searches.

Our final results analyze the incentives to provide information when several information providers compete for a favorable decision. A standard result in the literature holds that search activity and information provision increases when lobbyists compete for influence. We show that this result holds as long as interest groups do not have the ability to make contributions. When monetary incentives are allowed, the presence of a competing information provider reduces search, not only relative to the situation without contributions, but also relative to the situation without competing interests.

The analysis shows the relationship between two major interest group activities, campaign financing and information provision to political decision makers, which the literature has largely treated as separate issues. Our results suggest that the two activities are often strategic substitutes, as information inherently raises the price of contributions. In particular, we show that interest groups with intense preferences and high stakes in their preferred policy - extreme groups - strictly prefer offering contributions to the acquisition and provision of useful information. In this sense our analysis lends support to a rather cynical view of interest groups' role in the political process. 


\section{Appendix}

\section{Proof of Lemma 1}

Proof. If the lobby group searches but the search does not reveal any favorable information, the ex post contributions $c$ necessary to induce $d_{\ell}$ are

$$
\begin{aligned}
& c=\left[u_{d m}\left(d^{*} \mid m=\emptyset\right)-u_{d m}\left(d_{\ell} \mid m=\emptyset\right)\right] / g, \\
& \text { where } \quad d^{*}=\left\{\begin{array}{lll}
d_{\circ} & \Rightarrow u_{d m}\left(d^{*} \mid m=\emptyset\right)=-1 & \text { if } q<\frac{2}{3} \\
d_{r} & \Rightarrow u_{d m}\left(d^{*} \mid m=\emptyset\right)=\frac{1-q}{2-q}(-4) & \text { otherwise, }
\end{array}\right. \\
& \text { and } \quad u_{d m}\left(d_{\ell} \mid m=\emptyset\right)=\frac{-4}{2-q} \\
& \Rightarrow \quad c= \begin{cases}\frac{2+q}{(2-q) g} & \text { if } q<\frac{2}{3} \\
\frac{4 q}{(2-q) g} & \text { otherwise. }\end{cases}
\end{aligned}
$$

Notice that $u_{d m}$ is continuous in $q$, and that so is $c$.

Case $q<\frac{2}{3}$ : The lobby group prefers to induce $d_{\ell}$ instead of accepting $d_{\circ}$ if and only if

$$
\begin{array}{rlrl}
2 \beta-\frac{2+q}{(2-q) g} & >1 \\
\Leftrightarrow & \beta & >\frac{1}{2}+\frac{1+\frac{1}{2} q}{(2-q) g} .
\end{array}
$$

Case $q>\frac{2}{3}$ : The lobby group prefers to induce $d_{\ell}$ instead of $d_{r}$ if and only if

$$
\begin{array}{rlrl}
2 \beta-\frac{4 q}{(2-q) g} & >0 \\
\Leftrightarrow & \beta & >\frac{2 q}{(2-q) g} .
\end{array}
$$

The lobby group prefers to induce $d_{\ell}$ instead of inducing $d_{\circ}$ if and only if

$$
\begin{aligned}
2 \beta-\frac{4 q}{(2-q) g} & >1-\frac{1-4\left(\frac{1-q}{2-q}\right)}{g} \\
\Leftrightarrow \quad \beta & >\frac{1}{2}+\frac{1+\frac{1}{2} q}{(2-q) g} .
\end{aligned}
$$




\section{Proof of Result 2}

Proof. Assume that the decision the group wants to induce (using information and/or contributions) does not depend on the outcome of its search. We show that the expected value of searching and offering contributions ex post when the search does not reveal favorable information (i.e. $\sigma \neq \theta_{\ell}$ ) is less than the value of not searching and just offering contributions.

The "ex ante" contribution necessary to induce $d=d_{\ell}$ given the decision maker's prior beliefs is $\frac{1}{g}$. Thus, the value of offering contributions without searching is

$$
V_{\ell}(\text { contribution })=2 \beta-\frac{1}{g} .
$$

The ex ante value of searching and offering ex post contributions for the lobby group then becomes

$$
\begin{aligned}
V_{\ell}(\text { search }, \text { contribution }) & =2 \beta-\left(1-\frac{q}{2}\right) c-k \\
& = \begin{cases}2 \beta-\frac{2+q}{2 g}-k & \text { if } q<\frac{2}{3} \\
2 \beta-\frac{2 q}{g}-k & \text { if } q>\frac{2}{3},\end{cases}
\end{aligned}
$$

where $c$ is defined in equation (6).

The net expected gain from searching is therefore:

Gain from searching $=V_{\ell}($ search, contribution $)-V_{\ell}($ contribution $)$

$$
=\left\{\begin{aligned}
-\frac{2+q}{2 g}-k+\frac{1}{g}=\frac{-q}{2 g}-k & \text { if } q<\frac{2}{3} \\
-\frac{2 q}{g}-k+\frac{1}{g}=\frac{1-2 q}{g}-k & \text { if } q>\frac{2}{3} .
\end{aligned}\right.
$$

Notice that for $q>0$ the gain is strictly negative, even if $k=0$.

\section{Proof of Result 3}

Proof. Assume the decision maker expects the lobby group not to search. For this to be a rational expectation equilibrium, it must be the case that the value of searching followed by ex post contributions is less than the value of offering contributions alone, i.e.

$$
\frac{q}{2} 2 \beta+\left(1-\frac{q}{2}\right)\left(2 \beta-\frac{1}{g}\right)-k<2 \beta-\frac{1}{g}
$$


The right hand side is the value of offering contributions without searching. The equation reduces to the condition on $k$

$$
k>\frac{q}{2 g} \equiv \bar{k}
$$

Thus, if the direct cost of searching is sufficiently high, there exists a rational expectation equilibrium where the group does not search, and the contribution that induced $d=d_{\ell}$ is $\frac{1}{g}$.

Next, assume that the decision maker expects the lobby group to search. It was shown in the proof for Lemma 1 that the decision maker's expected utility of choosing $d_{\ell}$ conditional on $m=\emptyset$ is continuous in the success probability of the search $q$. Furthermore we showed there that the contribution necessary to induce $d_{\ell}$ when the decision maker expects the group to search is given by equation (6). In order for search to maximize the group's expected utility, the value of searching must exceed the value of not searching, or

$$
2 \beta-\left(1-\frac{q}{2}\right) c-k>2 \beta-c
$$

where $c$ is given in (6). Hence searching is a REE strategy if and only if

$$
k<\overline{\bar{k}} \equiv \begin{cases}\frac{q}{2 g}\left(\frac{2+q}{2-q}\right) & \text { if } q<\frac{2}{3} \\ \frac{q}{2 g}\left(\frac{4 q}{2-q}\right) & \text { otherwise. }\end{cases}
$$

\section{Mixed Strategies}

In this section we characterize the mixed strategy equilibria from Result 3.

Lemma 3 (Mixed strategies). Assume that search is unobservable, that the lobby group's preferences are sufficiently intense $(\beta>\underline{\beta}(q))$, and that $k \in[\bar{k}, \overline{\bar{k}}]$. Then there exists a mixed strategy rational expectation equilibrium that is characterized as follows. Define

$$
s_{1}=\frac{4 g k-2 q}{q^{2}+2 q g k} \quad \text { and } \quad s_{2}=\frac{2 g k}{2 q^{2}+q g k} .
$$


(i) If $q<\frac{2}{3}$, then the interest group searches with probability $s=s_{1}$. If it does not search or does not find favorable information, it offers contributions $C(s)=\frac{2+s q}{2-s q} / g$ for the choice of $d_{\ell}, 0$ otherwise.

(ii) If $q>\frac{2}{3}$, then the interest group searches with probability $s_{1}$ if $k \in\left[\bar{k}, k^{*}\right]$ and with probability $s_{2}$ if $k \in\left[k^{*}, \overline{\bar{k}}\right]$, where $k^{*}=\frac{q}{g}$. If it does not search or does not find favorable information, it offers contributions $C(s)=\frac{4 s q}{2-s q} / g$ for the choice of $d_{\ell}, 0$ otherwise.

In both cases the decision maker always chooses $d_{\ell}$ upon receiving hard evidence for $\theta_{\ell}$ or for contributions of at least $C(s)$.

Proof. Assume that the group uses a mixed search strategy in which it searches with probability $s$, and that the decision maker expects the group to search with probability $\tilde{s}$. Whenever the decision maker does not receive hard information $m=\theta_{\ell}$, she updates her beliefs about $\theta$, which becomes $p\left(\theta_{r} \mid m=\emptyset\right)=\frac{1 / 2}{1-\tilde{s} q / 2}=\frac{1}{2-\tilde{s} q}$. With this belief the optimal decision independently of contributions 'switches' at $\tilde{s} q=\frac{2}{3}$, i.e.

$$
d^{*}= \begin{cases}d_{\circ} & \text { if } \tilde{s} q<\frac{2}{3} \\ d_{r} & \text { otherwise. }\end{cases}
$$

The decision maker's expected utility from the project choice is

$$
u_{d m}\left(d^{*} \mid m=\emptyset\right)= \begin{cases}-1 & \text { if } \tilde{s} q<\frac{2}{3} \\ \frac{-4(1-\tilde{s} q)}{2-\tilde{s} q} & \text { otherwise, }\end{cases}
$$

while $u_{d m}\left(d_{\ell} \mid m=\emptyset\right)=\frac{-4}{2-\tilde{s} q}$. The contributions necessary to induce $d_{\ell}$ are therefore

$$
C(\tilde{s})=\left\{\begin{array}{lll}
\left(-1+\frac{4}{2-\tilde{s} q}\right) / g & =\frac{2+\tilde{s} q}{2-\tilde{s} q} / g & \text { if } \tilde{s} q<\frac{2}{3} \\
\left(\frac{-4(1-\tilde{s} q)}{2-\tilde{s} q}+\frac{4}{2-\tilde{s} q}\right) / g=\frac{4 \tilde{s} q}{2-\tilde{s} q} / g & \text { otherwise. }
\end{array}\right.
$$

In a mixed strategy rational expectations equilibrium the interest group must be indifferent between searching and not searching, or $\frac{q}{2} 2 \beta+\left(1-\frac{q}{2}\right)(2 \beta-$ $C(\tilde{s}))-k=2 \beta-C(\tilde{s})$, which implies

$$
\frac{q}{2} C(\tilde{s})=k .
$$


Furthermore, the expected search probability must equal the actual one, $\tilde{s}=s$.

Case 1: Assume $\tilde{s} q<\frac{2}{3}$. Plugging $C(\tilde{s})$ into (9), we get $\frac{q}{2} \frac{2+\tilde{s} q}{2-\tilde{s} q} / g=k$. Solving for the search probability yields

$$
\tilde{s}=\frac{4 g k-2 q}{q^{2}+2 q g k} \equiv s_{1}
$$

It is easy to show that $s_{1}$ is a valid probability $\left(s_{1} \in[0,1]\right)$ for $k \in[\bar{k}, \overline{\bar{k}}]$ (where $\bar{k}, \overline{\bar{k}}$ are defined in Result 3). Furthermore, $s_{1}$ is decreasing in $q$ and increasing in $k$ and $g$.

$q<\frac{2}{3}$ guarantees that $\tilde{s} q<\frac{2}{3}$. Thus, $\tilde{s}=s_{1}$ constitutes an equilibrium mixed strategy for the interest group and decision maker equilibrium belief whenever $q<\frac{2}{3}$ and $k \in[\bar{k}, \overline{\bar{k}}]$.

If $q>\frac{2}{3}$, then $\tilde{s} q<\frac{2}{3}$ requires that $\frac{4 g k-2 q}{q^{2}+2 q g k} q<\frac{2}{3}$. Solving for $k$ we get as condition for a valid probability

$$
k<\frac{q}{g} \equiv k^{*}
$$

It is easy to show that in this case $\bar{k}<k^{*}<\overline{\bar{k}}$. Thus, $\tilde{s}=s_{1}$ also constitutes an equilibrium mixed strategy for the interest group and decision maker equilibrium belief whenever $q>\frac{2}{3}$ and $k \in\left[\bar{k}, k^{*}\right]$.

Case 2: Assume $\tilde{s} q>\frac{2}{3}$. Plugging $C(\tilde{s})$ into (9) for this case we have $\frac{q}{2} \frac{4 \tilde{s} q}{2-\tilde{s} q} / g=k$. Solving for $\tilde{s}$ we get

$$
\tilde{s}=\frac{2 g k}{2 q^{2}+q g k} \equiv s_{2} .
$$

Again, one can show that $s_{2}$ is decreasing in $q$ and increasing in $k$ and $g$. For $k \in[0, \overline{\bar{k}}], s_{2}$ is a valid probability. Naturally, $\tilde{s} q>\frac{2}{3}$ implies $q>\frac{2}{3}$ and $k>k^{*}$. Thus, $\tilde{s}=s_{2}$ constitutes an equilibrium mixed strategy for the interest group and decision maker equilibrium belief whenever $q>\frac{2}{3}$ and $k \in\left[k^{*}, \overline{\bar{k}}\right]$. 


\section{References}

[1] Austen-Smith, David. "Interest Groups, Campaign Contributions, and Probabilistic Voting." Public Choice 54, 1987, pp. 123-39.

[2] Austen-Smith, David and John R. Wright. "Competitive Lobbying for a Legislator's Vote." Social Choice and Welfare 9, 1992, pp. 229-57.

[3] Austen-Smith, David. "Campaign Contributions and Access." American Political Science Review 89(3), 1995, pp. 566-81.

[4] Ball, Richard. "Interest Groups, Influence and Welfare." Economics and Politics 7(2), 1995, pp. 119-46.

[5] Banerjee, Abhijit. "A Simple Model of Voice." Conference on the Political Economy of Economics Policy, European University Institute: Florence, 1997, pp. 329-66.

[6] Baron, David P. "Service-induced Campaign Contributions and the Electoral Equilibrium." Quarterly Journal of Economics 104(1), 1989, pp. $45-73$.

[7] Baron, David P. "Electoral Competition with Informed and Uninformed Voters." American Political Science Review 88(1), 1994, pp. 33-47.

[8] Bernheim, B. Douglas and Michael D. Whinston. "Menu Auctions, Resource Allocation, and Economic Influence." Quarterly Journal of Economics, 1986. 101(1): pp. 1-31.

[9] Calvert, Randall L. "The Value of Information: A Rational Choice Model of Political Advice." Journal of Politics 47, 1985, pp. 530-55.

[10] Dewatripont, Mathias and Jean Tirole. "Advocates." Journal of Political Economy, 107(1), 1999, pp. 1-39.

[11] Gilligan, Thomas W. and Keith Krehbiel. "Collective Decisionmaking and Standing Committees: An Informational Rationale for Restrictive Amendment Procedures." Journal of Law, Economics and Organization 3(2), 1987, pp. 287-335.

[12] Grossman, Gene and Elhanan Helpman. "Protection for Sale." American Economic Review 84(4), 1994, pp. 833-50.

[13] Grossman, Gene and Elhanan Helpman. "Electoral Competition and Special Interest Politics." Review of Economic Studies 63, 1996, pp. 265-80. 
[14] Lohmann, Susanne. "Information, Access, and Contributions: A Signaling Model of Lobbying." Public Choice 85, 1995, pp. 267-84.

[15] Maskin, Eric and Jean Tirole. "Unforeseen Contingencies, Property Rights, and Incomplete Contracts." Review of Economic Studies.

[16] Persson, Torsten. "Economic Policy and Special Interest Politics." Economic Journal, forthcoming, 1998.

[17] Schlozman, Kay Lehman and John Tierney. Organized Interests and American Democracy. New York: Harper Collins, 1987.

[18] Snyder, James M. "Campaign Contributions As Investments: The U.S. House of Representatives, 1980-1986." Journal of Political Economy 98(6), 1990, pp. 1195-1227.

[19] Snyder, James M. "On Buying Legislatures." Economics and Politics 3(2), 1991, pp. 93-109. 